\title{
Instruction-based response activation depends on task preparation
}

\author{
Baptist Liefooghe • Jan De Houwer • Dorit Wenke
}

Published online: 15 February 2013

(C) Psychonomic Society, Inc. 2013

\begin{abstract}
An increasing number of studies have demonstrated that a response in one task can be activated automatically on the basis merely of instructed stimulus-response (S-R) mappings belonging to another task. Such instruction-based response activations are considered to be evidence for the formation of S-R associations on the basis of the S-R mappings for an upcoming, but not yet executed, task. A crucial but somewhat neglected assumption is that instructed $\mathrm{S}-\mathrm{R}$ associations are formed only under conditions that impose a sufficient degree of task preparation. Accordingly, in the present study we investigated the relation between task preparation and the instruction-based task-rule congruency effect, which is an index of response activation on the basis of instructions. The results from two experiments demonstrated that merely instructed S-R mappings of a particular task only elicit instruction-based response activations when that task is prepared for to a sufficient degree. Implications are discussed for the representation of instructed S-R mappings in working memory.
\end{abstract}

Keywords Task switching - Executive control · Attentional control $\cdot$ Working memory

In analogy to practiced stimulus-response (S-R) associations (Sudevan \& Taylor, 1987), S-R associations that are only formed on the basis of instructed S-R mappings can also lead to automatic response activations when they are task irrelevant (De Houwer, Beckers, Vandorpe, \& Custers,

\footnotetext{
B. Liefooghe $(\bowtie) \cdot J$. De Houwer

Department of Experimental-Clinical and Health Psychology,

Ghent University, Henri Dunantlaan 2,

9000 Ghent, Belgium

e-mail: baptist.liefooghe@ugent.be

D. Wenke

Humboldt University, Berlin, Germany
}

2005; Liefooghe, Wenke, \& De Houwer, 2012; Wenke, Gashler, \& Nattkemper, 2007). Both types of associations, however, are represented by different working memory components. Working memory supposedly exists through the combination of activated long-term memory (ALTM) and the direct-access region (DA; Oberauer, 2009). ALTM encompasses the activated representations of existent information, and DA consists of a restricted subset of highly activated representations within ALTM. While DA is capacity limited, ALTM is (virtually) not. Instructed S-R associations are assumed to be represented in DA in the form of a temporal binding (Hommel, 2009) between preexisting representations in ALTM, such as stimulus and response codes (Liefooghe et al., 2012; Meiran, Cole, \& Braver, 2012). In contrast, practiced S-R associations would be represented in ALTM, because these associations have been preestablished through practice (e.g., Meiran \& Kessler, 2008). This distinction is supported by studies demonstrating that instruction-based response activations can be eliminated by a concurrent working memory load (Meiran \& CohenKdoshay, 2012), while response activation on the basis of practiced S-R associations cannot (Kessler \& Meiran, 2010). Furthermore, response activation on the basis of practiced S-R associations is not affected by task preparation (Yamagushi \& Proctor, 2011), which suggests that such activation is related to the episodic retrieval of S-R associations from ALTM and is independent of top-down processes, such as maintenance in the DA.

In contrast, instruction-based response activations are assumed to only be present when the instructed task is prepared (Liefooghe et al., 2012; Meiran et al., 2012). Liefooghe et al. used the instruction-based task-rule congruency effect (IBTRCE) for measuring instruction-based response activations. Two tasks were used: an inducer task and a diagnostic task (Fig. 1). Each run started with the presentation of a pair of S-R mappings of the inducer task, which indicated responses to the 


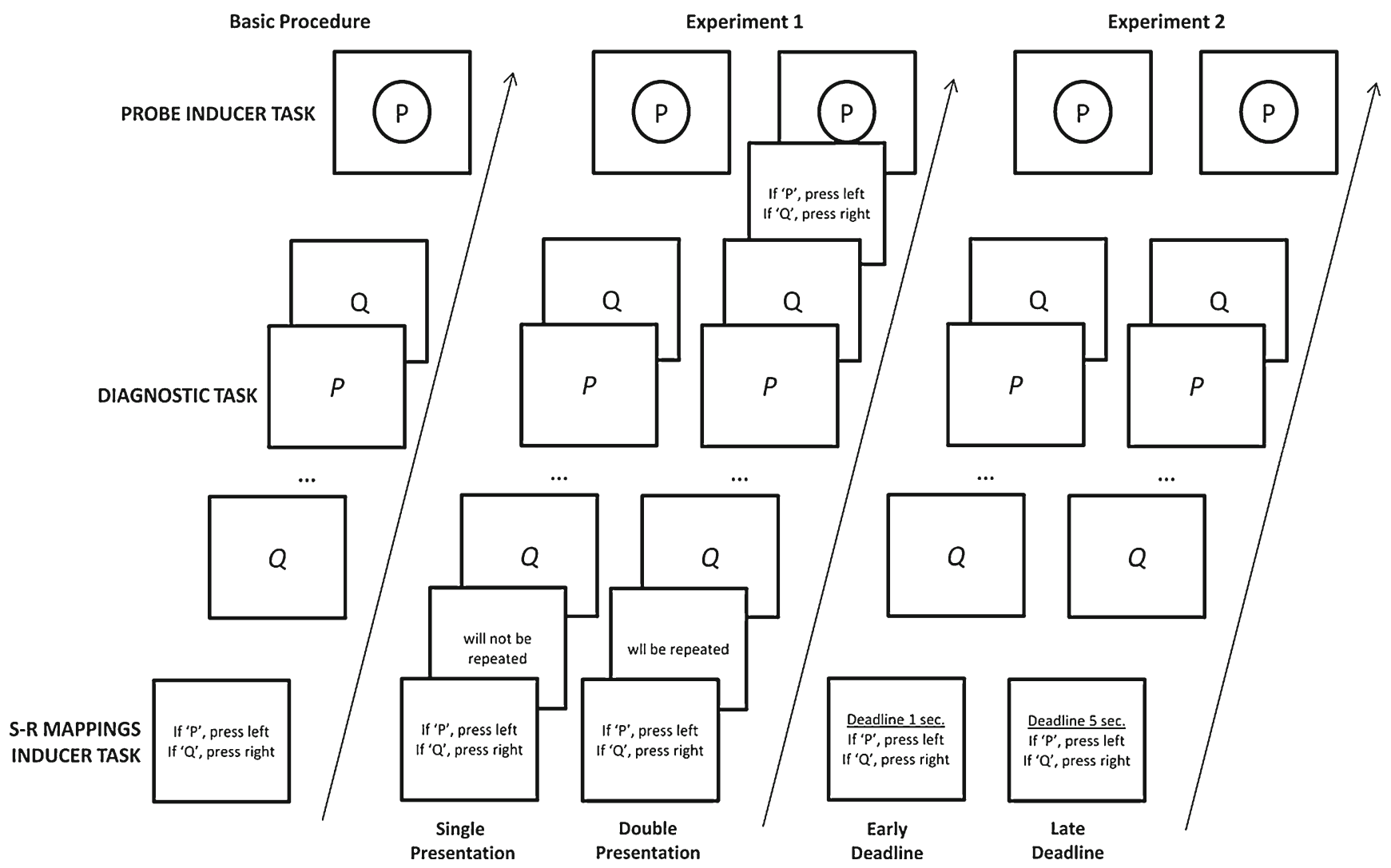

Fig. 1 Outline of the basic procedure used to measure the instruction-based task-rule congruency effect (IB-TRCE), along with the variants used in Experiments 1 and 2 of the present study

identity of a probe stimulus that would be presented later on. Between the onset of the S-R mappings and the onset of the probe, several trials of the diagnostic task were presented. Both tasks shared stimuli and responses, but now participants only responded to the orientation of the stimuli ("if upright, press left; if italic, press right"). The IB-TRCE refers to the finding that reaction times (RTs) in the diagnostic task were faster when the response in the diagnostic task corresponded to the S-R mapping for that stimulus in the inducer task (e.g., an upright "P" requiring a left keypress) than when it did not (e.g., an italic " $P$ " requiring a left keypress). The IB-TRCE was only present, however, when participants intended to apply one of the instructed S-R mappings. When the instructed $\mathrm{S}-\mathrm{R}$ mappings had to be maintained for future recall, no IB-TRCE was observed. Liefooghe et al. proposed that only when participants have to apply the S-R mappings of the inducer task this task is prepared for by making a compound of the relevant features needed for its execution, which elicits an IB-TRCE when this compound is irrelevant to the current task. Although task preparation was not directly manipulated, the study of Liefooghe et al. thus offered a first indication that instruction-based response activation and task preparation are related.

Wenke, Gaschler, Nattkemper, and Frensch (2009) likewise suggested that instruction-based response activation depends on task preparation. They used an inducer task, in which only one trial of a diagnostic task was embedded. In this diagnostic task, two adjacent letters with different font sizes were presented, and participants judged whether the bigger letter appeared on the left or on the right side of the screen center by pressing a central response key once or twice. RTs in the diagnostic task were slower when the letter position on the screen was incompatible with the response locations assigned to these letters in the instructed S-R mappings than when the left-right positions were compatible with the response locations of the instructed S-R mappings. On the basis of the assumption that the instructed response-location codes of the inducer task and the stimulus-location codes of the diagnostic task are represented in a common medium (Hommel, 2009), Wenke et al. (2009) concluded that this compatibility effect was related to instruction-based response activations. Interestingly, this effect was not present when no-go cues frequently signaled that the inducer task would not proceed. The frequent presentation of no-go cues may thus have discouraged participants from preparing for the inducer task, which again suggests that instruction-based response activation and task preparation are related. However, Meiran et al. (2012) argued that the compatibility effect observed by Wenke et al. (2007; Wenke et al., 2009) does not reflect instructionbased response activations, but the (mis)match between the 
left-right display of the letters in the diagnostic task and the S$\mathrm{R}$ mappings of the inducer task.

Although the studies of Wenke et al. (2009) and Liefooghe et al. (2012) suggest that instruction-based response activation depends on task preparation, their interpretation may be open for discussion. Accordingly, in the present study we go beyond these studies by offering a more unequivocal demonstration that instruction-based response activation depends on task preparation. To this end, we manipulated the necessity to prepare for the inducer task, while measuring whether this influenced the presence of an IB-TRCE in the diagnostic task.

\section{Experiment 1}

On each run of Experiment 1 (Fig. 1), a cue was presented immediately after having encoded the instructed S-R mappings of the inducer task, which indicated that these mappings either would reappear prior to the onset of the probe of the inducer task (double-presentation run) or would not (single-presentation run). For single-presentation runs, participants had to prepare for the inducer task in advance by forming S-R associations on the basis of the instructed S-R mappings. In contrast, on double-presentation runs, there was no need to do so, because the critical S-R mappings were represented a second time, and $\mathrm{S}-\mathrm{R}$ associations could be formed after the diagnostic task. If instruction-based response activation depends on task preparation, then the IB-TRCE should be larger or only present on singlepresentation runs.

\section{Method}

Participants A group of 30 students at Ghent University participated as a course requirement or for credit. All of the participants had normal or corrected-to-normal vision, and all were naïve to the purpose of the experiment.

Materials A list of 48 stimuli consisted of letters, numbers, and symbols. For each participant, a set of 24 pairs of stimuli was randomly constructed on the basis of this list. These pairs were randomly assigned to four blocks. The six pairs of each block were randomly assigned to the six runs within each block (two runs with four trials of the diagnostic task, two runs with eight diagnostic trials, and two runs with 16 diagnostic trials). For each run length, one run was a single-presentation run, and the other was a doublepresentation run. Each pair of stimuli was only used for one run. Run length varied randomly, so that the probe onset of the inducer task was unpredictable.

In each run, the inducer task and the diagnostic task used the same pair of stimuli and left-right keys ("A" and "P" on an AZERTY keyboard). In the inducer task, participants responded to a probe that was surrounded by a circle on the basis of the instructed $\mathrm{S}-\mathrm{R}$ mappings. In the diagnostic task, participants decided whether a stimulus (presented without a circle) was printed upright or in italic. The leftright response assignments in the diagnostic task were counterbalanced across participants.

On single-presentation runs, the instructed S-R mappings were presented only once, at the beginning of each run. On double-presentation runs, these $\mathrm{S}-\mathrm{R}$ mappings were also presented prior to the onset of the probe of the inducer task. The run type was cued immediately after the initial presentation of the instructed S-R mappings. This was done to ensure that participants still encoded the $\mathrm{S}-\mathrm{R}$ mappings on the double-presentation runs. The cues consisted of the messages wordt herhaald ("will be repeated") or wordt niet herhaald ("will not be repeated"), indicating whether the instructions would or would not be repeated. Finally, while the initial S-R mappings were always presented in black, the cueing message and the remainder of the run were presented in either blue or green, with each color indicating whether the participants were in a single- or doublepresentation run. The color-to-condition assignments were also counterbalanced across participants.

The stimuli for both tasks were presented at the center of a white screen in 36-point Arial font. S-R mappings and cue messages were presented in 16-point Arial font. The instructed $\mathrm{S}-\mathrm{R}$ mappings were randomly presented one above the other at the screen center, such that a mapping referring to a specific response key could be on either the top or the bottom line.

Procedure Participants were tested individually by means of a personal computer with a 17-in. color monitor running Tscope (Stevens, Lammertyn, Verbruggen, \& Vandierendonck, 2006). Instructions were presented on screen and paraphrased subsequently. Four blocks of six randomly ordered runs were presented, with a small break after each block. During each break, feedback was provided about the proportions of errors made on the diagnostic task and the inducer task. Each run started with the presentation of the S-R mappings of the inducer task. These mappings remained on screen until participants pressed the spacebar or until a maximum time of $10 \mathrm{~s}$ had elapsed. Once the mapping screen had disappeared, a cue was presented for $500 \mathrm{~ms}$ indicating whether the S-R mappings would or would not be repeated. The first stimulus of the diagnostic task was presented $500 \mathrm{~ms}$ after this cue. Each stimulus in the diagnostic task remained on screen until participants responded or until a maximum response time of 2,000 ms had elapsed. Depending on the run length, participants performed four, eight, or 16 trials of the diagnostic task, with an intertrial interval of $500 \mathrm{~ms}$. For single-presentation runs, $500 \mathrm{~ms}$ after the last response of the diagnostic task, the probe stimulus of the inducer task appeared. For double-presentation runs, 
$500 \mathrm{~ms}$ after the last response of the diagnostic task, the instructed S-R mappings of the inducer task were represented. As for the initial presentation, the S-R mappings remained on screen until participants pressed the spacebar or until $10 \mathrm{~s}$ had elapsed, after which the probe of the inducer task was presented. The probe remained on screen until participants responded or until a maximum response time of 2,000 ms had elapsed. A new run started after 1,500 ms. For incorrect responses, the screen turned red for $200 \mathrm{~ms}$. The experiment lasted for approximately $30 \mathrm{~min}$.

Results

Eight participants who failed to attain $70 \%$ of accuracy in the inducer task were considered outliers. The first block was considered practice and not analyzed. The means and standard deviations of all variables in the present study are presented in Table 1.

Diagnostic task Only runs on which the inducer task was performed correctly were considered. The first trial of each run was a switch trial and was not included. For the RTs, only correct trials were included. For each condition and each participant, RTs more than two standard deviations above the mean were removed (3.9\%). RTs were then logtransformed for the analyses. The RTs and accuracies were each subjected to a 2 (congruency) by 2 (run type: single or double presentation) repeated measures analysis of variance (ANOVA). RTs were longer on incongruent than on congruent trials, $F(1,21)=8.82, M S E=.001663, p<.01, \eta_{\mathrm{p}}{ }^{2}=$ .30. The main effect of run type was not significant, $F<1$. Both variables interacted, $F(1,21)=4.35, M S E=.001692$, $p<.05, \eta_{\mathrm{p}}{ }^{2}=.17$. For the single-presentation runs, RTs on incongruent trials were longer than RTs on congruent trials, $t(21)=4.14, p<.001, r^{2}=.45$, but this was not the case for double-presentation runs, $t<1$. For error rates, the effects of congruency and run type, as well as their interaction, were not significant. The largest $F$ value was observed for congruency: $F(1,21)=2.53, M S E=.00388, p=.127, \eta_{\mathrm{p}}{ }^{2}=.11$.

Inducer task Three separate ANOVAs, each with Run Type as a repeated measures factor, were conducted on the logtransformed encoding times (i.e., the time between the onset of the S-R mappings and participants' presses on the spacebar), log-transformed decision times (i.e., the time needed for responding to the probe), and decision errors. Encoding times of the first instruction presentation did not vary with run type, $F<1$. The decision times were longer for singlethan for double-presentation runs, $F(1,21)=160.19, M S E=$ $.0148, p<.001, \eta_{\mathrm{p}}{ }^{2}=.88$. Fewer decision errors were made on double- than on single-presentation runs, $F(1,21)=$ 24.10, MSE $=.006159, p<.001, \eta_{\mathrm{p}}^{2}=.53$.

\section{Discussion}

An IB-TRCE was present in the diagnostic task for single- but not for double-presentation runs. This suggests that on singlepresentation runs, participants prepared for the inducer task, which elicited instruction-based response activations in the diagnostic task. For double-presentation runs, participants possibly did not prepare for the inducer task, since the instructed S$\mathrm{R}$ mappings of the inducer task were represented prior to the probe onset of the inducer task, and no IB-TRCE was observed.

However, the results of the inducer task in Experiment 1 do not offer indications that participants actually prepared

Table 1 Mean results and the corresponding standard deviations (in parentheses) of Experiments 1 and 2

\begin{tabular}{|c|c|c|c|c|c|}
\hline & \multirow[t]{2}{*}{ Trial Type } & \multicolumn{2}{|l|}{ Experiment 1} & \multicolumn{2}{|l|}{ Experiment 2} \\
\hline & & Single Presentation & Double Presentation & Early Deadline & Late Deadline \\
\hline \multicolumn{6}{|c|}{ Diagnostic Task } \\
\hline \multirow[t]{3}{*}{ RTs } & Incongruent & $563(56)$ & $551(54)$ & $599(78)$ & $608(60)$ \\
\hline & Congruent & $539(58)$ & $547(50)$ & $571(67)$ & $599(72)$ \\
\hline & $I B-T R C E$ & 24 & 4 & 28 & 9 \\
\hline \multirow[t]{3}{*}{ Error rates } & Incongruent & $.09(.05)$ & $.09(.07)$ & $.08(.06)$ & $.07(.04)$ \\
\hline & Congruent & $.06(.06)$ & $.07(.05)$ & $.04(.04)$ & $.06(.07)$ \\
\hline & $I B-T R C E$ & .03 & .02 & .04 & .01 \\
\hline \multicolumn{6}{|c|}{ Inducer Task } \\
\hline \multicolumn{2}{|c|}{ Encoding times } & $3,822(2,042)$ & $3,760(2,118)$ & $5,689(3,663)$ & $5,177(2,942)$ \\
\hline \multicolumn{2}{|c|}{ Decision times } & $893(131)$ & $566(121)$ & 840 (157) & 914 (209) \\
\hline \multicolumn{2}{|c|}{ Decision error rates } & $.14(.10)$ & $.02(.06)$ & $.14(.14)$ & $.09(.09)$ \\
\hline
\end{tabular}

The size of the instruction-based task-rule congruency effect (IB-TRCE) is also reported. Note that untransformed reaction times (RTs), encoding times, and decision times are presented 
more thoroughly for single- than for double-presentation runs. This was prevented by the procedural parameters used in Experiment 1. First, the extent of preparation could not be estimated on the basis of the time to encode the initial instructions, simply because the cue about the repetition of instructions was presented only after the initial instructions had been encoded. Hence, there was no reason to assume that initial encoding times would differ between the two types of runs. Second, performance on the inducer task was also uninformative, because on double-presentation runs, the S-R mappings were represented immediately prior to the onset of the inducer task. The inducer task was thus much easier on double- than on single-presentation runs. It is thus difficult to conclude that single-presentation runs were associated with more task preparation than were the double-presentation runs. Furthermore, participants may have started to prepare for the inducer task on both types of runs immediately after the instructed S-R mappings were presented. The resulting $S-R$ associations could then have been sustained or removed, depending on whether a singleor double-presentation run was cued. In the latter case, the absence of instruction-based response activation may have been caused by the inhibition of the instructed S-R associations on double-presentation runs. The possible presence of such inhibition on those runs makes it unclear whether encoding instructed S-R mappings without actually preparing for them leads to instruction-based response activations.

\section{Experiment 2}

In view of the concerns associated with Experiment 1, in Experiment 2 we aimed to manipulate the necessity to prepare for the inducer task in a different way (see Fig. 1). On each run, participants were cued that they would have to respond to the probe of the inducer task within a $1,500-\mathrm{ms}$ response window (early-deadline run) or within a 5,000-ms response window (late-deadline run). The rationale was that participants should prepare for the inducer task on earlydeadline runs but not on late-deadline runs (see also Lien, Ruthruff, Remington, \& Johnston, 2005, for a similar logic). As such, S-R associations should be formed in advance for early- but not for late-deadline runs, and the IB-TRCE should be larger or only present on early-deadline runs. The deadline was now cued simultaneously with the instructed S-R mapping, and late-deadline runs thus did not trigger redundant task preparation, as had been the case on the double-presentation runs in Experiment 1. Moreover, the deadline manipulation allowed us to assess differences in task preparation by comparing performance on the inducer task between early- and late-deadline runs. A potential concern is that performance on the inducer task could be biased by a speed-accuracy trade-off, which is a common finding when using deadline procedures (Wickelgren, 1977). This would not apply for the diagnostic task (and thus for the IB-TRCE), in which the response deadline was not varied and participants had no reason to adopt different speed-accuracy strategies.

\section{Method}

A group of 34 participants were recruited from the same pool. None of them had participated in Experiment 1. The tasks and materials were the same as in Experiment 1, except for the following changes. First, only singlepresentation runs were used. Second, the response deadline to the probe of the inducer task was manipulated. On earlydeadline runs, the response deadline was $1,500 \mathrm{~ms}$, while on late-deadline runs, the deadline was $5,000 \mathrm{~ms}$. Run type was cued simultaneously with the presentation of the S-R mappings. Participants were cued that they had $1 \mathrm{~s}$ to respond for early-deadline runs and $5 \mathrm{~s}$ for late-deadline runs. For earlydeadline runs, the instructed deadline was thus shorter than the actual deadline. We did this in order to minimize the number of late responses, while at the same time maximizing the effort that participants would put into preparing the inducer task. Both run types were presented in blue or green, to inform participants about the ruling deadline.

\section{Results}

The exclusion criteria and raw-data processing were the same as in Experiment 1, resulting in the exclusion of eight participants.

Diagnostic task RTs (3.7 \% outliers) and accuracies were each subjected to a 2 (congruency: congruent or incongruent) by 2 (run type: early or late deadline) repeated measures ANOVA. RTs were longer on incongruent than on congruent trials, $F(1,25)=6.25, M S E=.004059, p<.05, \eta_{\mathrm{p}}{ }^{2}=.20$, and were shorter on early- than on late-deadline runs, $F(1,25)=$ $6.91, M S E=.004196, p<.05, \eta_{\mathrm{p}}{ }^{2}=.22$. The two main effects interacted, $F(1,25)=4.40, M S E=.0012375, p<.05, \eta_{\mathrm{p}}{ }^{2}=$ .15: For early-deadline runs, RTs were longer on incongruent than on congruent trials, $t(25)=3.59, p<.01, r^{2}=.34$, but this was not the case for late-deadline runs, $t(25)=1.07, p=.294$, $r^{2}=.04$.

For error rates, the main effect of congruency was significant, $F(1,25)=5.49, M S E=.002123, p<.05, \eta_{\mathrm{p}}{ }^{2}=.18$, with fewer errors on congruent than on incongruent trials. The main effect of run type was not significant, $F<1$. The interaction between the two main effects did not reach significance for errors, $F(1,25)=2.03, M S E=.004791, p=.17, \eta_{\mathrm{p}}{ }^{2}=.07$.

Inducer task We observed a trend toward shorter encoding times for late- as compared to early-deadline runs, $F(1,25)=$ 
3.26, MSE $=.027169, p=.083, \eta_{\mathrm{p}}{ }^{2}=.12$. Decision times were shorter for early- than for late-deadline runs, $F(1,25)=4.59$, $M S E=.017316, p<.05, \eta_{\mathrm{p}}{ }^{2}=.16$, and decision errors did not vary with run types, $F(1,25)=2.62, M S E=.010950, p=.118$, $\eta_{\mathrm{p}}^{2}=.09$.

\section{Discussion}

The IB-TRCE was present for early- but not for latedeadline runs. Performance in the inducer task also differed between the two run types. Encoding times were (numerically) shorter on late- than on early-deadline runs, and participants thus tended to spend less time processing $\mathrm{S}-\mathrm{R}$ mappings on late-deadline runs. Decision times on the inducer task were longer for late than for early deadlines. This pattern of results suggests that participants prepared for the inducer task more thoroughly on early-deadline runs than they did on late-deadline runs. Experiment 2 thus offers more direct evidence that the IB-TRCE depends on task preparation and that simply encoding instructed S-R mappings does not trigger an IB-TRCE.

Nevertheless, a main effect of run type was present, indicating that RTs of the diagnostic task were overall longer on late- than on early-deadline runs. Hence, instruction-based response activations may have started to decay on slow responses (Hommel, 1994) of late-deadline runs. To test this hypothesis, additional analyses were conducted in which response speed was taken into account by performing a median split of the RTs of the diagnostic task and including Response Speed as a factor. The interaction between response speed and congruency was not significant, $F(1,25)=2.42, M S E=$ $.0025512, p=.133, \eta_{\mathrm{p}}{ }^{2}=.09$, nor was the interaction between congruency, run type, and response speed, $F(1,25)=1.16$, $M S E=.0011376, p=.291, \eta_{\mathrm{p}}{ }^{2}=.04$. Finally, no IB-TRCE was present on the fastest responses of late-deadline runs, $t(25)=$ $1.05, p=.30, r^{2}=.04$. The IB-TRCE thus does not seem to have depended on response speed in the diagnostic task.

\section{General discussion}

In the present study, we tested whether instruction-based response activations depend on task preparation. We manipulated the amount of preparation that the inducer task required and measured the IB-TRCE in the diagnostic task. An IB-TRCE were only present when the S-R mappings of the inducer task were only presented once, at the beginning of a run (Exp. 1), and when an early deadline was imposed for the inducer task (Exp. 2). Experiment 2 also indicated different degrees of task preparation in the inducer task between early- and latedeadline runs, strengthening the conclusion that instructionbased response activation depends on task preparation.
The present experiments offer more evidence for the hypothesis that instruction-based response activations and task preparation are strongly related (Liefooghe et al., 2012; Meiran et al., 2012; Wenke et al., 2009), which suggests that instructions-based response activation depends on the active and advance implementation of instructed S-R mappings. Such implementation requires the temporary binding of conceptual codes representing the instructed stimuli and responses (Liefooghe et al., 2012; Wenke et al., 2007; Wenke et al., 2009), which may be a function of DA in working memory, by connecting stimulus and response representations in ALTM on the basis of the task instructions (Meiran et al., 2012). DA is thus involved only before actual responding is required when stringent preparation demands are imposed. When preparatory demands are absent or too lenient, DA is not involved beforehand, but only when the imperative stimulus is presented and the instructed $\mathrm{S}-\mathrm{R}$ mappings need to be implemented into functional $\mathrm{S}-\mathrm{R}$ associations in order to produce a response.

Instructed S-R mappings can thus be maintained without being implemented. This can possibly be explained by distinguishing between declarative and procedural working memory (Oberauer, 2009), in which declarative working memory holds representations for processing and procedural working memory holds representations that control processing, with each system having separate capacity limits. Previous research (Liefooghe et al., 2012; Wenke et al., 2009) suggested that instructed S-R mappings are maintained in declarative working memory, while implemented S-R associations are represented in procedural working memory, with only the latter type of representation eliciting instruction-based response activations. Alternatively, it is possible that $\mathrm{S}-\mathrm{R}$ mappings that have not yet been implemented are simply represented in ALTM and that S-R associations are only formed by means of the DA when necessary.

A puzzling finding in this context is that in both Experiments 1 and 2, RTs in the diagnostic task did not increase when (more) preparation was required in the inducer task. This suggests that being prepared for the inducer task does not elicit a larger load on the diagnostic task. The absence of such a load effect may suggest that maintaining nonimplemented S-R mappings in declarative working memory or maintaining implemented $\mathrm{S}-\mathrm{R}$ associations in procedural working memory delays processing in the diagnostic task to the same extent. Alternatively, it could be that performance in the diagnostic task in itself operates relatively independently of DA. The overly practiced S-R mappings of the diagnostic task may result in the formation of S-R associations in ALTM that are automatically retrieved when performing the diagnostic task. As such, the presence of additional S-R associations in DA may only minimally delay processing in the diagnostic task. Clearly, these issues indicate that future research will have to pinpoint the representational differences between implemented and nonimplemented S-R mappings. 
Taken together, our results are in line with the idea that instruction-based response activations depend on controlled, and likely strategically mediated, processes of advance preparation (Liefooghe et al., 2012; Meiran et al., 2012; Wenke et al., 2009) that result in the formation of S-R associations that automatically trigger responses, even when these associations are irrelevant for the current task. The present study thus again suggests that although both instructed and practiced S-R associations lead to automatic response activations, they are represented in different ways in working memory, with instructed S-R associations being more dependent on DA activity than are practiced $\mathrm{S}-\mathrm{R}$ associations.

Author note This research was supported by Grant No. BOF09/ 01M00209 from Ghent University to J.D.H.

\section{References}

De Houwer, J., Beckers, T., Vandorpe, S., \& Custers, R. (2005). Further evidence for the role of mode-independent short-term associations in spatial Simon effects. Perception \& Psychophysics, 67, 659-666. doi:10.3758/BF03193522

Hommel, B. (1994). Spontaneous decay of response code activation. Psychological Research, 56, 261-268. doi:10.1007/BF00419656

Hommel, B. (2009). Action control according to TEC (theory of event coding). Psychological Research, 73, 512-526. doi:10.1007/ s00426-009-0234-2

Kessler, Y., \& Meiran, N. (2010). The reaction-time task-rule congruency effect is not affected by working memory load: Further support for the activated long-term memory hypothesis. Psychological Research, 74, 388-399.

Liefooghe, B., Wenke, D., \& De Houwer, J. (2012). Instructionbased task-rule congruency effects. Journal of Experimental Psychology: Learning, Memory, and Cognition, 38, 13251335.
Lien, M.-C., Ruthruff, E., Remington, R. W., \& Johnston, J. C. (2005). On the limits of advance preparation for a task switch: Do people prepare all the task some of the time or some of the task all the time? Journal of Experimental Psychology. Human Perception and Performance, 31, 299-315. doi:10.1037/0096-1523.31.2.299

Meiran, N., \& Cohen-Kdoshay, O. (2012). Working memory load but not multitasking eliminates the prepared reflex: Further evidence from the adapted flanker paradigm. Acta Psychologica, 139, 309313. doi:10.1016/j.actpsy.2011.12.008

Meiran, N., Cole, M. W., \& Braver, T. S. (2012). When planning results in loss of control: Intention-based reflexivity and working-memory. Frontiers in Human Neuroscience, 6, 104. doi:10.3389/fnhum.2012.00104

Meiran, N., \& Kessler, Y. (2008). The task rule congruency effect in task switching reflects activated long-term memory. Journal of Experimental Psychology. Human Perception and Performance, 34, 137-157. doi:10.1037/0096-1523.34.1.137

Oberauer, K. (2009). Design for a working memory. In B. H. Ross (Ed.), The psychology of learning and motivation: Advances in research and theory (Vol. 51, pp. 45-100). San Diego: Academic Press. doi:10.1016/S0079-7421(09)51002-X

Stevens, M., Lammertyn, J., Verbruggen, F., \& Vandierendonck, A. (2006). Tscope: A C library for programming cognitive experiments on the MS Windows platform. Behavior Research Methods, 38, 280-286. doi:10.3758/BF03192779

Sudevan, P., \& Taylor, D. A. (1987). The cuing and priming of cognitive operations. Journal of Experimental Psychology. Human Perception and Performance, 13, 89-103.

Wenke, D., Gaschler, R., \& Nattkemper, D. (2007). Instructioninduced feature binding. Psychological Research, 71, 92-106. doi:10.1007/s00426-005-0038-y

Wenke, D., Gaschler, R., Nattkemper, D., \& Frensch, P. A. (2009). Strategic influences on implementing instructions for future actions. Psychological Research, 73, 587-601. doi:10.1007/ s00426-009-0239-x

Wickelgren, W. A. (1977). Speed-accuracy tradeoff and information processing dynamics. Acta Psychologica, 41, 67-85. doi:10.1016/ 0001-6918(77)90012-9

Yamaguchi, M., \& Proctor, R. W. (2011). Automaticity without extensive training: The role of memory retrieval in implementation of task-defined rules. Psychonomic Bulletin \& Review, 18, 347-354. doi:10.3758/s13423-011-0050-8 